\title{
Résultats et Perspectives de l'Assurance Vie en Italie
}

\author{
par Antonio Longo *
}

De 1973 à 1979, le secteur de l'assurance-vie a traversé en Italie l'une des périodes les plus mouvementées de son histoire.

L'indice 100, concernant la recette des primes des Compagnies en 1973, est passé à 193,5 en 1979 , ce qui traduit une augmentation annuelle de $11,63 \%$. Cependant, si l'on tient compte de l'inflation au cours de la même période, cet indice est passé de 100 en 1973 à 77,2 en 1979 , ce qui revient à dire que la perte réelle en portefeuille est de $22,8 \%$.

\begin{tabular}{|c|c|c|c|c|c|c|c|c|}
\hline & \multicolumn{2}{|c|}{$\begin{array}{c}\text { ASSURANCES } \\
\text { INDIVI- } \\
\text { DUELLES }\end{array}$} & \multicolumn{2}{|c|}{$\begin{array}{l}\text { ASSURANCES } \\
\text { COLLECTIVES }\end{array}$} & \multicolumn{2}{|c|}{ TOTAL } & \multicolumn{2}{|c|}{ INFLATION } \\
\hline & $\begin{array}{l}\text { Milliards/ } \\
\text { lires }\end{array}$ & indice & $\begin{array}{l}\text { Milliards/ } \\
\text { lires }\end{array}$ & indice & $\begin{array}{l}\text { Milliards/ } \\
\text { lires }\end{array}$ & indice & $\begin{array}{c}\operatorname{taux} a \\
\%\end{array}$ & indice \\
\hline 1973 & 271,5 & $100,-$ & 114,9 & $100,-$ & 386,4 & $100,-$ & 10,27 & 100 \\
\hline 1974 & 314,3 & 115,8 & 137,6 & 119,8 & 451,9 & $117,-$ & 19,44 & 119 \\
\hline 1975 & 386,2 & 142,2 & 157,7 & 137,2 & 543,9 & 140,8 & 17,17 & 140 \\
\hline 1976 & 445,5 & 164,1 & 197,6 & $172,-$ & 643,1 & 166,4 & 16,52 & 163 \\
\hline 1977 & $471,-$ & 173,5 & 215,4 & 187,5 & 686,4 & 177,6 & 18,10 & 192 \\
\hline 1978 & 513,1 & $189,-$ & 176,5 & 153,6 & 689,6 & 178,5 & 12,45 & 216 \\
\hline 1979 & 560,3 & 206,4 & 187,5 & 163,2 & 747,8 & 193,5 & 15,74 & 250 \\
\hline
\end{tabular}

Les budgets de toutes les compagnies et de tous les intermédiaires (agents et producteurs) pour qui les coûts fixes ont augmenté dans une proportion supérieure au taux de l'inflation s'en sont durement ressenti, et le secteur a aussitôt, dans tout son ensemble, donné des graves signes de crise.

Diverses raisons expliquent cette évolution :

- le blocage en 1977 de l'échelle mobile sur l'indemnité d'ancienneté. Cette mesure a eu directement de lourdes conséquences sur les affaires des assurances collectives ;

- toujours en 1977, la loi fiscale, visant à éviter les abus connus par le passé, a fini par pénaliser l'épargne d'assurance.

* Président de l'INA - Institut National des Assurances, Rome. 
Mais la cause la plus déterminante, celle qui a eu la plus grande influence, a sans aucun doute été l'inflation. En face d'elle, les compagnies semblaient ne pas parvenir à réagir de façon appropriée. Elles se sont ainsi laissé entraîner dans la spirale d'un accroissement permanent de l'indexation des coûts de gestion, trop important par rapport au niveau des portefeuilles. Si l'on a enregistré des tentatives de réaction, c'était dans des secteurs marginaux d'activité et elles n'étaient pas en mesure d'offrir une solution à la crise. En 1977, la base de mortalité des tarifs temporaires en cas de mort a été réajustée, ce qui a donné lieu à une relance positive, compte tenu du fait qu'il s'agissait des seuls tarifs qui n'imposent pas de plafond à l'assureur, et lui permettent de protéger l'assuré contre l'inflation.

En 1978, les principales compagnies ont émis un tarif rente-viager immédiat qui garantissait une indexation annuelle de la rente sur $50 \%$ du taux de l'inflation. Mais, comme ce tarif s'adressait à un secteur particulier du marché et parce qu'il était à prime unique, ce tarif n'a pas pu ramener le volume des activités dans la branche assurance-vie au niveau qu'aurait exigé la baisse de l'ensemble des affaires.

En effet, les polices d'assurance à prime annuelle avec un fort contenu-épargne, le produit le plus solide et le plus traditionnel de l'assurance-vie (même dans l'assurance collective), se sont heurtées à la résistance croissante du public. Le fait est que les compagnies ont des difficultés à offrir par ce type de polices des gains qui soient d'une part capables de suivre le taux de l'inflation, et d'autre part comparables aux gains offerts par d'autres formes d'investissement. Les taux d'inflation élevés ont également provoqué de profondes mutations économiques et financières, et, partant, dans tout le secteur de l'assurance-vie.

La période a été marquée par une forte tendance à l'épargne familiale, qui a connu des creux pendant les années de forte hausse des prix et des pointes lorsque l'inflation reculait, "parce que les épargnants cherchaient à reconstituer la valeur de leurs richesses financières » (Rapport du Gouverneur de la Banque d'Italie - 1978 - page 301).

Les dépôts à vue et titres à court terme ont attiré ces mouvements d'épargne : "le recours à de tels titres permet d'ajuster les rendements nominaux aux variations des deux indicateurs du taux d'inflation, sans indexation 》 (ibidem - page 372). Cependant, ces variations se sont avérées de plus en plus nécessaires pour essayer d'orienter, dans l'intérêt de l'économie nationale, les mouvements d'épargne vers des investissements, financiers ou non, à plus long terme. En fait, les plans de relance du gouvernement, dans le bâtiment, destinés à résoudre la crise du logement, prévoyaient l'indexation des titres et des prêts fonciers (plan décennal et épargne-logement). Certains de ces plans étaient déjà entrés en phase d'application concrète. L'introduction de l'« equo canone »1, visant à rééquilibrer les situations passées, avait explicitement exprimé la nécessité de lier les bénéfices des capitaux immobilisés à long terme aux variations du coût de la vie. En outre, des formes d'indexation financière ont été introduites sur le marché («prime rate » et taux d'intérêt des obligations).

2. Vers la fin de 1979, l'Institut National des Assurances a reçu l'autorisation d'émettre une nouvelle police d'assurance, la « Monnaie Forte »». Il s'agit d'une assurance mixte de rente à prime annuelle différée. Son but était de protéger les prestations

1 « Equo canone : Loi no 392 du 27.7 .78 sur la fixation des loyers (note du traducteur). 
offertes de l'inflation. Du point de vue pratique, pour qu'une assurance-vie à prime annuelle puisse défendre, de façon juste et équitable, le pouvoir d'achat de l'épargne de l'assuré, il faut que les deux parties concernées, l'assureur et l'assuré, contribuent dans la mesure de la charge qui leur revient.

Par exemple, l'assuré doit ajuster les primes qu'il s'est engagé à verser à l'avenir aux variations du pouvoir d'achat de la monnaie. Quant à l'assureur, il doit pouvoir garantir une réévaluation adéquate de ce que l'assuré lui versera au cours du contrat.

La nouvelle police d'assurance « Monnaie Forte » prévoit l'indexation de la prime et du taux d'intérêt réel sur la variation du coût de la vie (50\% de l'inflation $+3 \%)$. Ainsi, l'indexation "contractuelle » du capital et de la rente sur la variation des prix est garantie.

Du point de vue économique, ce qui sous-tend l'obligation de la part de l'assuré d'indexer les primes, c'est la capacité des travailleurs à défendre la valeur réelle de leurs propres revenus. Outre le fait qu'elle est l'élément technique indispensable à l'ajustement du capital, l'indexation des primes offre également à l'assureur une source indexée pour financer ses frais de gestion.

De son côté, l'assureur peut s'acquitter de son engagement de réévaluer les réserves obtenues en utilisant ses réserves de façon à permettre une telle réévaluation. Le marché italien, comme on l'a déjà dit, offrait, vers la fin des années soixante-dix, des possibilités d'emploi où l'indexation sur l'inflation ou sur des paramètres financiers était explicite, comme par exemple avec le «prime rate», ou implicite, comme dans le cas des bons du trésor à court terme, car leur taux était constamment ajusté en fonction du marché et donc indirectement ajusté sur le taux d'inflation. Cependant, avec la police d'assurance "Monnaie Forte », l'INA s'engageait à garantir des gains indexés sur les primes que l'assuré aurait versées. Deux éléments sous-tendent cette politique.

Le premier, théorique : On s'est rendu compte que, dans une situation où le taux d'inflation reste élevé pendant plusieurs années, les taux d'intérêt tendent à suivre le niveau de l'inflation. L'expérience italienne de ces dix dernières années confirme ce principe théorique.

Le second, pratique: L'accord entre l'INA et l'ENEL (Société Nationale pour l'Energie Electrique) a été reconduit. Selon cet accord, l'ENEL s'engageait à mettre à disposition pendant trente ans 2.500 milliards de lires en obligations dont le coupon était basé sur le taux d'inflation. Emboîtant le pas de l'INA, toutes les sociétés ont renouvelé leurs tarifs d'assurance-mixte et de rente, non seulement en transformant le mécanisme d'indexation sur le coût de la vie adopté par l'Institut, mais également en explorant d'autres voies. La solution proposée par les sociétés participait du principe traditionnel de la distribution « a posteriori » des bénéfices de gestion, tout en l'enrichissant d'un engagement contractuel à un niveau minimal de participation. Les nouveaux contrats prévoyaient, en particulier, une gestion financière indépendante des réserves et l'attribution aux assurés d'une quote-part, non inférieure à $70 \%-80 \%$ du rendement annuel de la gestion.

Dans la plupart des cas, on demande à l'assuré d'ajuster les primes, et avec l'ajustement du bénéfice des réserves, on obtient ainsi l'ajustement du capital dans la même proportion. 
Il s'agit bien sûr d'un système qui diffère de la « Monnaie Forte », mais l'objectif final est le même : garantir la valeur réelle des capitaux assurés. Les résultats des premières années d'activité montrent que les objectifs visés par les assureurs sont atteignables.

Le public a très bien réagi. En 1980, l'INA a plus que doublé les primes de première année pour les assurances individuelles (dans un premier temps, les nouvelles polices d'assurance ne touchaient que ce secteur). En 1981, les nouvelles polices d'assurance ont suivi la même évolution.

Les portefeuilles en ont immédiatement bénéficié et ont retrouvé leur valeur réelle, malgré la persistance du taux élevé d'inflation.

$\begin{array}{lccc} & \text { Recette des primes de police individuelle } & & \frac{\text { Inflation }}{\text { (indice) }} \\ 1979 & 100,- & 100,- \\ 1980 & 117,5 & 120,5 \\ 1981 & 141,6 & 143,- \\ 1982 & 2170,- & 166,5\end{array}$

Le secteur des polices d'assurance collectives en Italie, lié, du moins jusqu'à aujourd'hui, à l'Institut pour l'indemnité d'ancienneté, est toujours en crise. La nouvelle réglementation de l'indemnité de retraite (qui indexe, en outre, les cotisations annuelles sur les variations du coût de la vie), ainsi que l'introduction de nouveaux tarifs dont les buts sont les mêmes que ceux recherchés dans le secteur des assurances individuelles, devraient offrir une possibilité de relance soutenue dans ce secteur. L'avenir de ce secteur, où la différence entre le marché italien et celui des autres pays plus développés économiquement et du point de vue de l'assurance, se fait le plus sentir, dépend du rôle que le législateur assignera aux retraites complémentaires au côté de la prévoyance obligatoire. Nous y reviendrons plus tard.

Une autre police d'assurance INA est la dernière nouveauté en matière tariffaire (approuvée fin 1982 et en phase de lancement) : elle est liée à la valeur des parts d'un fonds d'investissement « interne " au patrimoine de l'Institut. Nouveauté absolue sur le marché italien qui s'inspire des méthodes adoptées avec succès depuis quelques années sur le marché britannique. C'est également le signe de la vitalité de notre marché qui pourra explorer d'autres voies dans cette direction.

Le «tournant », provoqué par les assureurs dans la gestion du secteur assurancevie depuis 1979, a touché, comme on l'a vu, l'élément épargne inhérent aux assurances de type mixte. L'objectif recherché a été de relancer la police d'assurance-vie sous forme d'épargne.

Pour la distinguer de l'épargne traditionnelle, au sens étroit, on pourrait la définir en deux mots comme épargne-assurance.

2 Estimation sur recette jusqu'à septembre. 
On entend, par là, le prélèvement systématique effectué, pendant un certain temps, sur le revenu des personnes actives, au cours de leur vie active, en vue de financer les besoins du ménage au cours de la période d'inactivité, c'est-à-dire lorsque le revenu se réduit ou disparaît.

L'épargne-assurance diffère de l'épargne au sens étroit du terme, car du point de vue institutionnel, elle tient compte de la possibilité de perte ou réduction de revenu, survenue brutalement pendant la période d'activité, en cas d'invalité ou décès.

Autre caractère fondamental de cette forme d'épargne, qui la différencie des autres et qui a de grandes retombées sur l'économie : l'engagement contractuel, librement consenti, de versements des primes. Ainsi c'est la consommation de l'épargnant qui revêt un caractère résiduel et non plus l'épargne. La fonction de l'épargne est donc transformée.

Les caractéristiques propres aux assurances-vie, en tant que formes d'épargne, nous aident à identifier plus facilement les conditions extérieures pour qu'elles puissent se développer et prospérer en Italie.

Tout d'abord, les contrats offerts par l'assureur doivent être en mesure de protéger la valeur réelle de l'épargne de l'assuré quel que soit l'instrument pratique adopté. Les assureurs italiens ont fait des pas décisifs dans cette direction.

Il existe pourtant des problèmes concernant la vente et l'efficacité des vendeurs : leur "professionnalisme " est devenu le facteur prépondérant du succès de ce secteur. L'image de l'intermédiaire responsable de la vente des polices d'assurance-vie doit refléter les qualités, requises tant du point de vue de la préparation que de celui de la spécialisation, par les caractéristiques de produits vendus. Le nombre élevé de démarchages permis par la qualité de ces polices est en mesure de supporter le prix économique que supposent les qualités professionnelles des intermédiaires. Cependant, sur le plan des lois en vigueur, d'autres mesures sont nécessaires. La collectivité nationale peut en bénéficier en termes de développement économique et de stabilité sociale, si le législateur affirme le rôle prépondérant de l'assurance-vie libre dans notre pays. Ce dernier doit lui accorder une attention toute particulière, chose que, malheureusement, il n'a pas suffisamment faite jusqu'à aujourd'hui.

Depuis quelques années, les assureurs cherchent à pallier les différences flagrantes de traitement : en effet, ceux qui perçoivent un revenu unique ne peuvent soustraire à la «source» du revenu imposable (dans le cadre de l'impôt sur le revenu) les primes versées pour l'assurance-vie. Ils n'obtiennent l'exonération prévue par la loi qu'en passant par le mécanisme de crédit à l'impôt, qui est long et onéreux. Il faut également réajuster le plafond du montant déductible qui a beaucoup souffert de l'érosion monétaire au cours de ces dernières années. Les facilités de paiements et les exonérations fiscales doivent constamment être sous le contrôle des gouvernants.

Lorsque, et c'est le cas pour l'assurance-vie, on ne remet pas en question l'opportunité de l'existence des facilités de paiements et des exonérations, le contrôle doit s'effectuer conformément à une loi claire et précise, sachant sauvegarder les objectifs essentiels, et non pas selon des mécanismes généralisés tels que le non-ajustement du plafond et la pénalisation d'un grand nombre de citoyens. Ces mécanismes ont souvent comme résultat celui de déformer radicalement les objectifs fixés. 
Sur le plan législatif, le problème le plus urgent et le plus grave que l'on rencontre, lorsqu'on essaie de tracer le cadre futur de l'assurance-vie en Italie, est celui de définir le rôle et de fixer les limites respectives de l'assurance obligatoire et de l'assurance volontaire. On ne peut séparer le problème de la réforme de la retraite en Italie, de certains choix primordiaux.

Il est inutile de disserter sur le fait de savoir si l'Italie s'est trompée plus ou moins que d'autres pays.

Ce qui est objectif et concret, c'est que la conjoncture économique a changé dans le monde entier et qu'il est de plus en plus indispensable de changer d'attitude vis-à-vis des problèmes d'assurance.

Les coûts de l'assurance obligatoire doivent être bloqués à leur niveau actuel. Les moyens disponibles doivent être répartis de manière plus équitable afin de garantir un niveau de vie minimal à ceux qui n'ont pas eu suffisamment de possibilités d'épargner pendant leur vie active. Les coûts supplémentaires que nous aurions obligatoirement et automatiquement à supporter, et que ne feraient qu'aggraver les automatismes destructeurs, pourraient éventuellement être rendus disponibles en vue d'opérations volontaires. Ainsi pourrait se créer un nouveau type d'assurances, réalisation qui serait rendue possible grâce à l'apport conjoint des travailleurs et des employeurs. Les avantages que tireraient les travailleurs de l'institutionalisation de ce système d'épargne-prévoyance sont évidents. Quant aux employeurs, ils trouveraient sans doute intéressant de pouvoir négocier ce qui, au contraire, leur serait débité dans un système d'indexation pratiquement automatique.

La couverture obligatoire et celle complémentaire devront être financées par la contribution conjointe des travailleurs et des employeurs. En revanche la troisième dépendra des disponibilités individuelles.

La gestion de la deuxième couverture se ferait selon des règles qui assurent constamment la capitalisation des intérêts rapportés afin de garantir la solvabilité du système. Les investissements à long terme en bénéficieront également. Les assureurs italiens du secteur de l'assurance-vie sont prêts à consacrer leurs efforts et leurs qualités professionnelles à l'élaboration d'un système d'assurance plus juste et plus efficace. 